MS24-03

\section{Investigation of planar defects in electrode materials using FAULTS}

Marine Reynaud ${ }^{1}$, Jon Serrano-Sevillano ${ }^{1}$, Amaia Saracibar ${ }^{2}$, Thomas Altantzis $^{3}$, Sara Bals ${ }^{3}$, Gustaaf van Tendeloo ${ }^{3}$, Jokin Rikarte ${ }^{1}$, Juan Rodríguez-Carvajal ${ }^{4}$, Montse Casas-Cabanas ${ }^{1}$

1. Solid State Electroactive Materials Group - CIC energiGUNE, Vitoria-Gasteiz, Spain

2. Physical Chemistry Department, Basque Country University, Pharmacy Faculty, Vitoria-Gasteiz, Spain

3. Electron Microscopy for Materials Research (EMAT), University of Antwerp, Antwerp, Belgium

4. Diffraction group - Institut Laue Langevin, Grenoble, France

email: mreynaud@cicenergigune.com

The physical-chemical properties of functional materials are directly related to their structural features, and this is particularly relevant in the field of electrochemical energy storage. The thorough microstructural characterization of these materials, which includes the determination of different kinds of defects, their amount and their locations, is therefore of high importance.

Most Rietveld refinement programs include microstructural models that enable to extract valuable information regarding microstructural features such as point defects, finite crystallite size, microstrains or antiphase domains. All these features have a strong impact on materials' properties and are therefore essential variables for their complete understanding. However this approach faces several limitations in the case of crystalline materials with extended defects such as stacking faults, since they are poorly described with an average unit cell.

A different approach, using the recursive property of crystals, was used to develop the DIFFaX code, which allows simulating diffraction patterns of crystal systems with coherent planar defects [1]. Taking a step further, we have developed the FAULTS program from the core of DIFFaX so as to enable the refinement of experimental X-ray and Neutron powder diffraction patterns of faulted crystal systems [2]. An improved version of this program, more performant and with additional features is now available as an open program (www.cicenergigune.com/faults) and within the FullProf suite of programs (www.ill.eu/sites/fullprof).

Our presentation will show examples of several battery materials having different kinds of defects to illustrate how, besides classic structural determination, precise quantitative information regarding microstructural features such as stacking faults, twinning or intergrowths can now be extracted from diffraction data to establish correlations with materials' properties. Hence, using a simple layered description for the 3D framework of EMD- $\mathrm{MnO}_{2}$, we performed the first quantitative analysis of ramsdellite motifs into a pyrolusite structure [2]. Another example refers to Li-rich layered oxides such as $\mathrm{Li}_{2} \mathrm{MnO}_{3}$, (also written $\mathrm{Li}\left[\mathrm{Li}_{1 / 3} \mathrm{Mn}_{2 / 3}\right] \mathrm{O}_{2}$ to better account for its layered structure). These materials are known to crystallize with significant amounts of stacking faults, and we will show how the FAULTS program has allowed decoupling the individual role of crystallite size and amount of defects on the electrochemical properties of a series of samples of $\mathrm{Li}_{2} \mathrm{MnO}_{3}$ [3].

Acknowledgements: This research work has received funding from the Spanish Ministerio de Economía y Com- petitividad (MINECO, projects ENE2016 81020 R, ENE2016 75242 R and grant FJCI-2014-19990) and from the Spanish Ministerio de Educación, Cultura y Deporte (MECD, grant CAS15/00354).

\section{References:}

[1] Treacy, M. M. et al. (1991). Proc. R. Soc. London Ser. A, 433, 499-520. doi: 10.1098/rspa.1991.0062.

[2] Casas-Cabanas, M. et al. (2016). J. Appl. Cryst., 49, 2259-2269. doi: $10.1107 / \mathrm{S} 1600576716014473$.

[3] Serrano-Sevillano, J. et al. Submitted.

Keywords: powder diffraction, stacking faults, battery materials 